\title{
Shock Wave Solutions for Some Nonlinear Flow Models Arising in the Study of a Non-Newtonian Third Grade Fluid
}

\author{
Taha Aziz, R. J. Moitsheki, A. Fatima, and F. M. Mahomed \\ Centre for Differential Equations, Continuum Mechanics and Applications, School of Computational and Applied Mathematics, \\ University of the Witwatersrand, Wits 2050, South Africa
}

Correspondence should be addressed to R. J. Moitsheki; raseelo.moitsheki@wits.ac.za

Received 28 February 2013; Revised 6 June 2013; Accepted 7 June 2013

Academic Editor: Waqar Khan

Copyright (C) 2013 Taha Aziz et al. This is an open access article distributed under the Creative Commons Attribution License, which permits unrestricted use, distribution, and reproduction in any medium, provided the original work is properly cited.

This study is based upon constructing a new class of closed-form shock wave solutions for some nonlinear problems arising in the study of a third grade fluid model. The Lie symmetry reduction technique has been employed to reduce the governing nonlinear partial differential equations into nonlinear ordinary differential equations. The reduced equations are then solved analytically, and the shock wave solutions are constructed. The conditions on the physical parameters of the flow problems also fall out naturally in the process of the derivation of the solutions.

\section{Introduction}

A shock wave is a disturbance that propagates through a media. Shock is yielded when a disturbance is made to move through a fluid faster than the speed of sound (the celerity) of the medium. This can occur when a solid object is forced through a fluid. It represents a sharp discontinuity of the parameters that delineate the media. Unlike solutions where the energy is a conserved quantity and thus remains constant during its propagation, shock wave dissipates energy relatively quickly with distance. One source of a shock wave is when the supersonic jets fly at a speed that is greater than the speed of sound. This results in the drag force on aircraft with shocks. These waves also appear in various interesting phenomena in real life situations. For example, solitons appear in the propagation of pulses through optical fibers. Another example is where cnoidal waves appear in shallow water waves, although this is an extremely scarce phenomena. Some interesting communications dealing with the shock wave solutions are found in [1-3].

During the past several decades, the study of the nonlinear problems dealing with the flow of non-Newtonian fluids has attracted considerable attention. This interest is due to several important applications in engineering and industry such as reactive polymer flows in heterogeneous porous media, electrochemical generation of elemental bromine in porous electrode systems, manufacture of intumescent paints for fire safety applications, extraction of crude oil from the petroleum products, synthetic fibers, and paper production [4]. Due to the diverse physical nature of nonNewtonian fluids, there is no single constitutive expression which describe the physical behavior of all non-Newtonian fluid models. Because of this important issue, several models of non-Newtonian fluids have been proposed in the literature. Together with this factor, the mathematical modelling of nonNewtonian incompressible fluid flows gives rise to nonlinear and complicated differential equations. As a consequence of this nonlinearity factor, the exact (closed-form) solutions of these sort of problems are scarce in general. Several techniques and methods have been developed in the recent few years to construct the solutions of these non-Newtonian fluid flow problems. Some of these useful methods are variational iteration method, Adomian decomposition method, homotopy perturbation method, homotopy analysis method, and semi-inverse variational method. Literature survey witnesses that, despite all these methods, the exact (closed-form) solutions of the non-Newtonian fluid flow problems are still rare in the literature, and it is not surprising that new exact (closed-form) solutions are most welcome, provided they correspond to physically realistic situations. Some interesting and useful communications in this area are made in the studies [5-14]. 


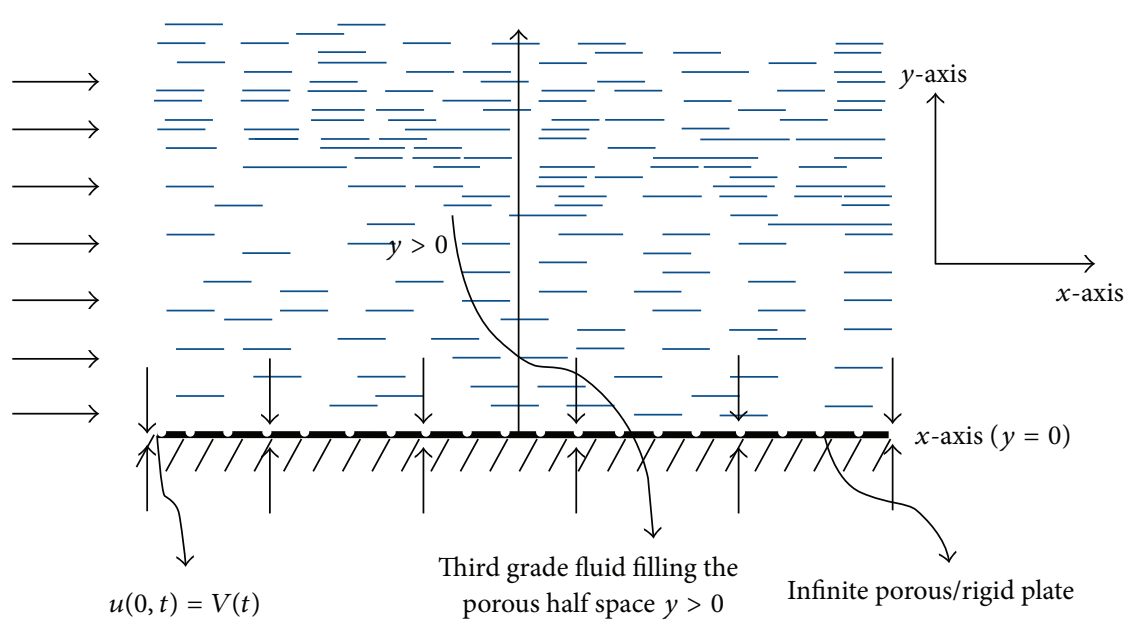

FIGURE 1: Geometry of the physical models and coordinate system.

One of the widely accepted models amongst nonNewtonian fluids is the class of Rivlin-Ericksen fluids of differential type [15]. Rivlin-Ericksen fluids of differential type have acquired the special status in order to describe the several nonstandard features such as normal stress effects, rod climbing, shear thinning, and shear thickening. In the literature much attention has been given to the flow of a second grade fluid [16-19]. A second grade fluid model is the simplest subclass of non-Newtonian fluids for which one can reasonably hope to obtain an analytical solution. In most of the flow situations, the governing equations for a second grade fluid are linear. Although a second grade fluid model is able to predict the normal stress differences, it does not take into account the shear thinning, and shear thickening phenomena that many fluids reveal. Therefore some experiments may be well described by third grade fluid [20-24]. The mathematical model of a third grade fluid represents a more realistic description of the behavior of nonNewtonian fluids. A third grade fluid model represents a further attempt towards the study of the flow properties of non-Newtonian fluids. Therefore, a third grade fluid model has been considered in this study. This model is known to capture the non-Newtonian effects such as shear thinning or shear thickening as well as normal stress.

In this particular study, we have constructed the shock wave solutions of some nonlinear PDEs arising in the study of third grade fluid flow in porous medium. We know that the flow of non-Newtonian fluids in a porous medium has wide range of engineering applications. These include enhanced oil recovery, ceramic processing, and geothermal engineering. Motivated by these facts, we have developed some shock wave solutions of three nonlinear problems dealing with the unsteady flow of third grade fluid in a porous half space.

\section{Geometry of the Models}

Consider a Cartesian coordinate frame of reference $O X Y Z$ with $x$-axis along the direction of the flow and $y$-axis pointing in the vertically upward direction. The third grade fluid occupies the porous space $y>0$ and is in contact with an infinite plate at $y=0$. Since the plate is infinite in the $X Z$ plane, therefore all the physical quantities except the pressure depend on $y$ only. We have taken three different problems on the same flat plate geometry. The geometry of the problems is shown in Figure 1.

\section{Problems to Be Investigated}

3.1. Unsteady Flow of a Third Grade Fluid over a Flat Rigid Plate with Porous Medium. Following the methodology of $[22,23]$, the unsteady incompressible flow of a third grade fluid over the rigid plate with porous medium is governed by

$$
\begin{aligned}
\left(\rho+\alpha_{1} \frac{\varphi}{\kappa}\right) \frac{\partial u}{\partial t}= & \mu \frac{\partial^{2} u}{\partial y^{2}}+\alpha_{1} \frac{\partial^{3} u}{\partial y^{2} \partial t}+6 \beta_{3}\left(\frac{\partial u}{\partial y}\right)^{2} \frac{\partial^{2} u}{\partial y^{2}} \\
& -2 \beta_{3} \frac{\varphi}{\kappa}\left(\frac{\partial u}{\partial y}\right)^{2} u-\frac{\varphi}{\kappa} \mu u,
\end{aligned}
$$

where $u$ is the velocity component in $x$-direction, $t$ is time, $y$ is spatial variable, $\rho$ is the density, $\mu$ is the coefficient of viscosity, $\alpha_{1}$ and $\beta_{3}$ are the material constants (for details on these material constants and the conditions that are satisfied by these constants, the reader is referred to [24]), $\varphi$ is the porosity and $\kappa$ is the permeability of the porous medium.

In order to solve (1), the relevant boundary and initial conditions are

$$
\begin{gathered}
u(0, t)=u_{0} g(t), \quad t>0, \\
u(\infty, t)=0, \quad t>0, \\
u(y, 0)=f(y), \quad y>0,
\end{gathered}
$$

where $u_{0}$ is the reference velocity and $g(t)$ and $f(y)$ are yet functions to be determined. The first boundary condition (2) is the no-slip condition, and the second boundary condition (3) says that the main stream velocity is zero. This is not a restrictive assumption since we can always measure velocity 
relative to the main stream. The initial condition (4) indicates initially that the fluid is moving with some nonuniform velocity $f(y)$.

3.1.1. Reduction of the Governing Equation. We know that from the principal of Lie symmetry methods $[25,26]$ that if a differential equation is explicitly independent of any dependent or independent variable, then this particular differential equation remains invariant under the translation symmetry corresponding to that particular variable. We noticed that (1) admits Lie point symmetry generators, $\partial / \partial t$ (translation in $t$ ) and $\partial / \partial y$ (translation in $y$ ). Let $X_{1}$ and $X_{2}$ be time-translation and space-translation symmetry generators, respectively. Then the solution corresponding to the generator

$$
X=X_{1}+m X_{2}=\frac{\partial}{\partial t}+m \frac{\partial}{\partial y}, \quad(m>0)
$$

would represent travelling wave solution with constant wave speed $m$. Travelling wave solutions are characterized by the fact that the profiles of these solutions at different time instants are obtained from one another by appropriate shifts (translations) along the $y$-axis. Consequently, a Cartesian coordinate system moving with the constant speed can be introduced in which the profile of the derived quantity is stationary. $m=0$ represents the stationary or steady-state solutions.

The characteristic system corresponding to (5) is

$$
\frac{d y}{m}=\frac{d t}{1}=\frac{d u}{0}
$$

Solving (6), invariant solutions are given by

$$
u(y, t)=F(\eta) \quad \text { with } \eta=y-m t
$$

where $F(\eta)$ is an arbitrary function of the characteristic variable $\eta=y-m t$. Making use of (7) into (1) results in a third-order ordinary differential for $F(\eta)$ as follows:

$$
\begin{aligned}
(\rho+ & \left.\alpha_{1} \frac{\varphi}{\kappa}\right) m \frac{d F}{d \eta}+\mu \frac{d^{2} F}{d \eta^{2}}-m \alpha_{1} \frac{d^{3} F}{d \eta^{3}} \\
& +6 \beta_{3}\left(\frac{d F}{d \eta}\right)^{2} \frac{d^{2} F}{d \eta^{2}}-2 \beta_{3} \frac{\varphi}{\kappa} F\left(\frac{d F}{d \eta}\right)^{2}-\mu \frac{\varphi}{\kappa} F=0 .
\end{aligned}
$$

Thus the original third-order nonlinear PDE (1) is reduced to a third-order ODE (5) along certain curves in the $y$ - $t$ plane. These curves are called characteristic curves or just the characteristic.

3.1.2. Shock Wave Solution. In this section, we show that the travelling wave solutions of (1) approach a shock wave solution. Now we construct the shock wave solution of the reduced equation (5). The starting hypothesis for shock wave solution is given by

$$
F(\eta)=a \exp (b \eta)
$$

where $a$ and $b$ are the free parameters to be determined. Substituting (9) into (8), we obtain

$$
\begin{gathered}
{\left[m\left(\rho+\alpha_{1} \frac{\varphi}{\kappa}\right) b+\mu b^{2}-m \alpha_{1} b^{3}-\mu \frac{\varphi}{\kappa}\right]} \\
+e^{2 b \eta}\left[6 \beta_{3} a^{2} b^{4}-2 \beta_{3} \frac{\varphi}{\kappa} a^{2} b^{2}\right]=0 .
\end{gathered}
$$

Separating (10) in the powers of $e^{0}$ and $e^{2 b \eta}$, we find

$$
\begin{gathered}
e^{0}: m\left(\rho+\alpha_{1} \frac{\varphi}{\kappa}\right) b+\mu b^{2}-m \alpha_{1} b^{3}-\mu \frac{\varphi}{\kappa}=0, \\
e^{2 B \eta}: \beta_{3} a^{2}\left[3 b^{4}-\frac{\varphi}{\kappa} b^{2}\right]=0, \quad \text { with } \beta_{3} a^{2} \neq 0 .
\end{gathered}
$$

From (12), we deduce

$$
b=\sqrt{\frac{\varphi}{3 \kappa}} .
$$

Using the value of $b$ in (11), we obtain

$$
m\left(\rho+\alpha_{1} \frac{\varphi}{\kappa}\right) \sqrt{\frac{\varphi}{3 \kappa}}+\mu\left(\frac{\varphi}{3 \kappa}\right)-m \alpha_{1}\left(\frac{\varphi}{3 \kappa}\right) \sqrt{\frac{\varphi}{3 \kappa}}-\mu \frac{\varphi}{\kappa}=0 .
$$

Finally, the solution for $F(\eta)$ (provided the condition (14) holds) is written as

$$
F(\eta)=a \exp \left[\sqrt{\frac{\varphi}{3 \kappa} \eta}\right] .
$$

So the solution $u(y, t)$ which satisfies the condition (14) is written as

$$
u(y, t)=a \exp \left[\sqrt{\frac{\varphi}{3 \kappa}}(y-m t)\right] \text { with } m>0 .
$$

Remark 1. Note that the solution (16) is the shock wave solution to the governing PDE (1). The previous solution is valid under the particular condition on the physical parameters of the flow given in (14) (which is some kind of dispersion relation in $m$ ). This solution does show the hidden shock wave behavior of the flow problem with slope of the velocity field or the velocity gradient approaches to infinity such that

$$
\frac{\partial u}{\partial y} \rightarrow \infty \quad \text { as } y \rightarrow \infty
$$

Remark 2. Note that the solution (16) also satisfies the particular initial and the boundary condition; that is,

$$
\begin{array}{ll}
u(0, t)=g(t)=\exp \left[-\sqrt{\frac{\varphi}{3 \kappa}} m t\right], & t>0, \\
u(y, 0)=f(y)=\exp \left[\sqrt{\frac{\varphi}{3 \kappa}} y\right], & y>0,
\end{array}
$$

with

$$
g(0)=f(0)=a=1 .
$$

The functions $g(t)$ and $f(y)$ depend on the physical parameters of the flow. 
Remark 3. We also observe that the physical significance of the imposing condition (14) is that it gives the speed of travelling shock wave. From (14), we deduce

$$
m=\frac{2 \mu(\varphi / 3 \kappa)^{1 / 2}}{\left(\rho+\left(2 \alpha_{1} \varphi / 3 \kappa\right)\right)}>0 .
$$

3.2. Unsteady Magnetohydrodynamic (MHD) Flow of Third Grade Fluid in a Porous Medium. By employing the same geometry as we have explained in Section 2, in this problem we extend the previous model by considering the fluid to be electrically conducting under the influence of a uniform magnetic field applied transversely to the flow. We provide the closed-form solution of the problem by reducing the governing nonlinear PDE into an ODE with the help of Lie reduction technique.

The time-dependent magnetohydrodynamic flow of a third grade fluid in a porous half space in the absence of the modified pressure gradient takes the form

$$
\begin{aligned}
\left(\rho+\alpha_{1} \frac{\varphi}{\kappa}\right) \frac{\partial v}{\partial t}= & \mu \frac{\partial^{2} v}{\partial y^{2}}+\alpha_{1} \frac{\partial^{3} v}{\partial y^{2} \partial t}+6 \beta_{3}\left(\frac{\partial v}{\partial y}\right)^{2} \frac{\partial^{2} v}{\partial y^{2}} \\
& -2 \beta_{3} \frac{\varphi}{\kappa}\left(\frac{\partial v}{\partial y}\right)^{2} u-\frac{\varphi}{\kappa} \mu v-\sigma B_{0}^{2} v
\end{aligned}
$$

where $v$ is the velocity component in $x$-direction, $\sigma$ is the electrical conductivity, and $B_{0}$ is the uniform applied magnetic field. In order to solve (21), the relevant time and space dependent velocity boundary conditions are

$$
\begin{gathered}
u(0, t)=u_{0} g(t), \quad t>0, \\
u(\infty, t)=0, \quad t>0, \\
u(y, 0)=f(y), \quad y>0 .
\end{gathered}
$$

As it can be seen (21) also admits Lie point symmetry generators, $\partial / \partial t$ (translation in $t$ ) and $\partial / \partial y$ (translation in $y$ ). Let $X_{1}$ and $X_{2}$ be time-translation and space-translation symmetry generators, respectively. The invariant solution corresponding to the generator $X=X_{1}+m X_{2}$ is given by

$$
v(y, t)=G(\xi) \quad \text { with } \xi=y-m t .
$$

Using (23) into (21) yields

$$
\begin{gathered}
\left(\rho+\alpha_{1} \frac{\varphi}{\kappa}\right) m \frac{d G}{d \xi}+\mu \frac{d^{2} G}{d \xi^{2}}-m \alpha_{1} \frac{d^{3} G}{d \xi^{3}}+6 \beta_{3}\left(\frac{d G}{d \xi}\right)^{2} \frac{d^{2} G}{d \xi^{2}} \\
-2 \beta_{3} \frac{\varphi}{\kappa} G\left(\frac{d G}{d \xi}\right)^{2}-\mu \frac{\varphi}{\kappa} G-\sigma B_{0}^{2} G=0 .
\end{gathered}
$$

Following the same methodology adopted to solve the previous problem, the reduced ODE (24) admits an exact solution of the form

$$
G(\xi)=a \exp \left[\sqrt{\frac{\varphi}{3 \kappa}} \xi\right]
$$

provided that

$$
\begin{aligned}
& m\left(\rho+\alpha_{1} \frac{\varphi}{\kappa}\right) \sqrt{\frac{\varphi}{3 \kappa}}+\mu\left(\frac{\varphi}{3 \kappa}\right) \\
& -m \alpha_{1}\left(\frac{\varphi}{3 \kappa}\right) \sqrt{\frac{\varphi}{3 \kappa}}-\mu \frac{\varphi}{\kappa}-\sigma B_{0}^{2}=0 .
\end{aligned}
$$

Thus the solution of the PDE (21) which satisfies the condition (26) is written as

$$
v(y, t)=\exp \left[\sqrt{\frac{\varphi}{3 \kappa}}(y-m t)\right] \text { with } m>0 .
$$

Remark 4. Note that the previous solution (27) also satisfies the boundary and initial conditions given in (22). The imposing physical condition (26) gives the speed of travelling shock wave. From (26), we find

$$
m=\frac{2 \mu(\varphi / 3 \kappa)+\sigma B_{0}^{2}}{(\varphi / 3 \kappa)^{1 / 2}\left[\rho+\left(2 \alpha_{1} \varphi / 3 \kappa\right)\right]}>0 .
$$

If we set $B_{0}=0$ (no magnetic field), we recover the condition given in (14).

3.3. Unsteady Magnetohydrodynamic (MHD) Flow of Third Grade Fluid in a Porous Medium with Plate Suction/Injection. This particular model is an extension of previous two problems with combined effects of plate suction/injection and MHD nature of the fluid. Thus, for flow under consideration, we seek a velocity of the form

$$
\mathbf{V}=[w(y, t),-W, 0]
$$

where $w$ denotes the velocity of the fluid in $x$-direction and $W>0$ indicates suction velocity and $W<0$ blowing or injection velocity.

The unsteady MHD flow of a third grade fluid in a porous half space with plate suction/injection is governed by

$$
\begin{aligned}
\left(\rho+\alpha_{1} \frac{\varphi}{\kappa}\right) \frac{\partial w}{\partial t}= & \left(\rho+\alpha_{1} \frac{\varphi}{\kappa}\right) W \frac{\partial w}{\partial y}+\mu \frac{\partial^{2} w}{\partial y^{2}}+\alpha_{1} \frac{\partial^{3} w}{\partial y^{2} \partial t} \\
& +6 \beta_{3}\left(\frac{\partial w}{\partial y}\right)^{2} \frac{\partial^{2} w}{\partial y^{2}}-\alpha_{1} W \frac{\partial^{3} w}{\partial y^{3}} \\
& -2 \beta_{3} \frac{\varphi}{\kappa}\left(\frac{\partial w}{\partial y}\right)^{2} u-\frac{\varphi}{\kappa} \mu w-\sigma B_{0}^{2} w .
\end{aligned}
$$

The PDE (30) is solved subject to the same boundary and initial conditions specified for the previous models. The invariant solution of the previous nonlinear PDE under the linear combination of time-translation and space-translation symmetry generators is given by

$$
w(y, t)=H(\theta) \quad \text { with } \theta=y-m t .
$$


Inserting (31) in (30), we get a third-order nonlinear ODE in $H(\theta)$, namely,

$$
\begin{array}{r}
\left(\rho+\alpha_{1} \frac{\varphi}{\kappa}\right) m \frac{d H}{d \theta}+\left(\rho+\alpha_{1} \frac{\varphi}{\kappa}\right) W \frac{d H}{d \theta}+\mu \frac{d^{2} H}{d \theta^{2}} \\
-m \alpha_{1} \frac{d^{3} H}{d \theta^{3}}+6 \beta_{3}\left(\frac{d H}{d \theta}\right)^{2} \frac{d^{2} H}{d \theta^{2}}-\alpha_{1} W \frac{\partial^{3} H}{\partial \theta^{3}} \\
-2 \beta_{3} \frac{\varphi}{\kappa} H\left(\frac{d H}{d \theta}\right)^{2}-\left(\mu \frac{\varphi}{\kappa}+\sigma B_{0}^{2}\right) H(\theta)=0 .
\end{array}
$$

Following the same procedure used to tackle the first problem, (32) admits the exact solution of the form

$$
H(\theta)=a \exp \left[\sqrt{\frac{\varphi}{3 \kappa}} \theta\right]
$$

provided

$$
\begin{aligned}
0= & m\left(\rho+\alpha_{1} \frac{\varphi}{\kappa}\right) \sqrt{\frac{\varphi}{3 \kappa}}+\left(\rho+\alpha_{1} \frac{\varphi}{\kappa}\right) W \sqrt{\frac{\varphi}{3 \kappa}}+\mu\left(\frac{\varphi}{3 \kappa}\right) \\
& -(m+W) \alpha_{1}\left(\frac{\varphi}{3 \kappa}\right) \sqrt{\frac{\varphi}{3 \kappa}}-\left(\mu \frac{\varphi}{\kappa}+\sigma B_{0}^{2}\right) .
\end{aligned}
$$

The solution of $w(y, t)$ is written as

$$
w(y, t)=\exp \left[\sqrt{\frac{\varphi}{3 \kappa}}(y-m t)\right] \quad \text { with } m>0 .
$$

Remark 5. The previous solution is valid only under the particular condition on the physical parameters given in (34). The condition (34) also gives the speed of shock wave. Thus from (34), we obtain

$$
m=\frac{2 \mu(\varphi / 3 \kappa)+\sigma B_{0}^{2}-\left(\rho+\left(\alpha_{1} \varphi / 3 \kappa\right)\right) \sqrt{\varphi / 3 \kappa} W}{\sqrt{\varphi / 3 \kappa}\left[\rho+\left(2 \alpha_{1} \varphi / 3 \kappa\right)-\left(\alpha_{1} \varphi / 3 \kappa\right) W\right]}>0 .
$$

Note that, if we set $W=B_{0}=0$ (with no porosity and magnetic field), we recover the previous two conditions given in (14) and (26).

Remark 6. We note that the shock wave solutions (16), (27), and (35) are the same, but the imposing conditions on the physical parameters of the flow given in (14), (26), and (34) under which these solutions are valid are different. This means that in each case the speed of the travelling shock wave is different. Therefore, the graphical behavior of these solutions is the same which shows the shock wave behavior of the flow problems. However, the imposing conditions contain the magnetic field, suction/blowing, porosity, and second grade and the third grade parameters. Thus these closed-form shock wave solutions are valid for the particular values of these parameters.

\section{Shock Wave Behavior of the Solutions}

Figures 2 and 3 show the shock wave behavior of the solutions (16), (27), and (35) in $2 \mathrm{D}$ and $3 \mathrm{D}$, respectively. From the

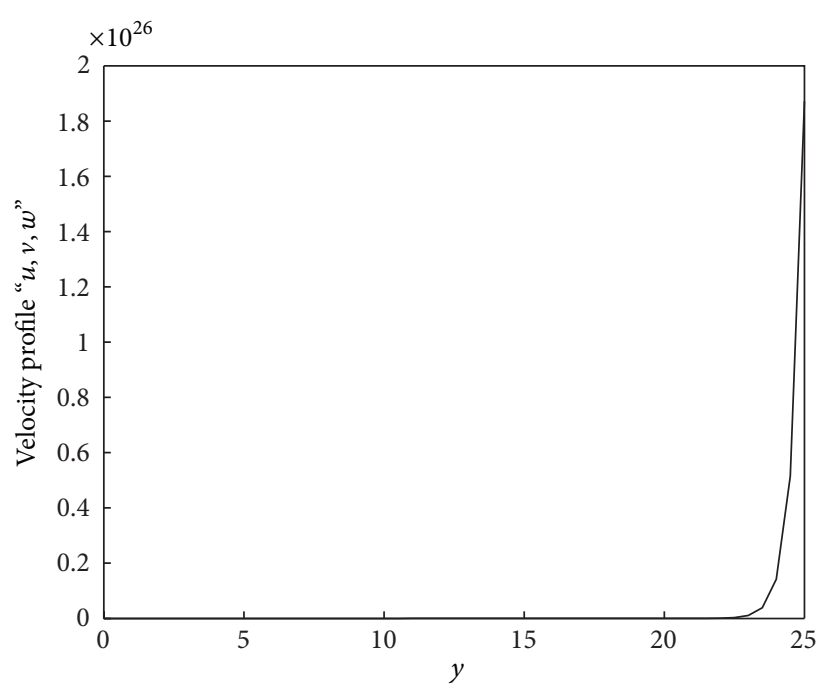

Figure 2: Profile of the shock wave solutions (16), (27), and (35) with $\varphi=4, \kappa=0.2, m=1$, and $t=\pi / 2$.

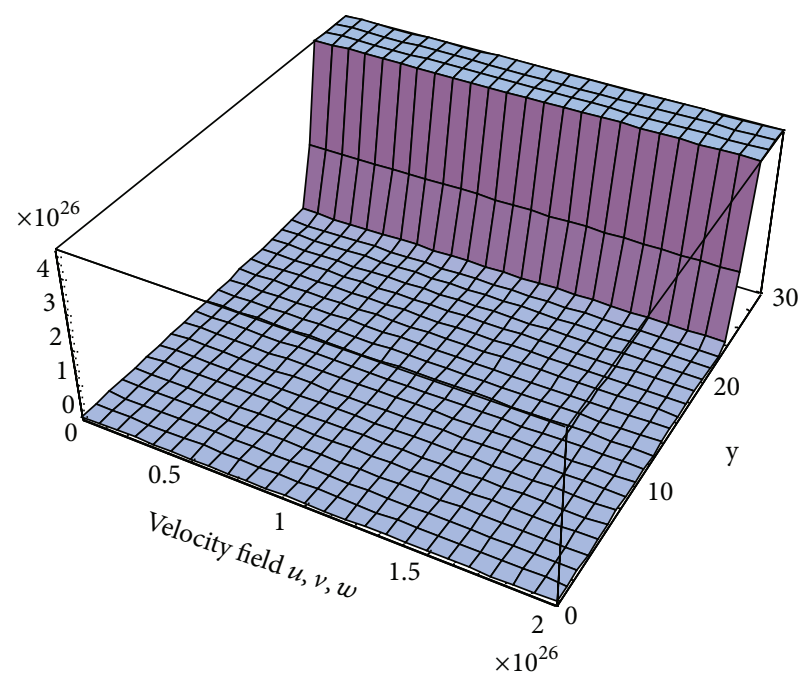

Figure 3: 3D profile of the shock wave solutions (16), (27), and (35) with $\varphi=4, \kappa=0.2, m=1$, and $t=\pi / 2$.

graphs it is quite clear that the slope of the velocity profile is approaching to infinity representing the sudden sharp discontinuity in the velocity field.

\section{Final Comments}

In this paper, we have presented closed-form shock wave solutions for some nonlinear problems which describe the phenomena of third grade fluids. In each case the governing nonlinear PDEs are reduced to nonlinear ODEs by using the Lie point symmetry (which is translation) in the $t$ and $y$ directions. The reduced ODEs are then solved analytically. We observe that the shock wave solutions (16), (27), and (35) are the same, but the imposing conditions on the physical parameters of the flow models given in (14), (26), and (34) under which these solutions are valid are different. These solutions 
do not directly contain the parameter which is responsible for showing the behavior of third grade fluid parameter on the flow. However, the imposing conditions under which these solutions are valid do contain the third grade parameter. To emphasize, we can say that these solutions are valid for the particular values of third grade fluid parameter. The results obtained describe the mathematical structure of the shock wave behavior of the flow problems. The models considered in this study are prototype, but the obtained solutions are going to be very helpful in carrying out further analysis of the shock wave characteristic associated with the non-Newtonian fluid flow models. The method that we have adopted is also prosperous for tackling wide range of nonlinear problems in non-Newtonian fluid mechanics.

\section{Acknowledgments}

T. Aziz and A. Fatima would like to thank the School of Computational and Applied Mathematics and the Financial Aid and Scholarship office, University of the Witwatersrand, for financial support and scholarships.

\section{References}

[1] T. Aziz, A. Fatima, and F. M. Mahomed, "Shock wave solution for a nonlinear partial differential equation arising in the study of a non-Newtonian fourth grade fluid model," Mathematical Problems in Engineering, vol. 2013, Article ID 573170, 5 pages, 2013.

[2] Y.-Z. Peng, "Exact solutions for some nonlinear partial differential equations," Physics Letters A, vol. 314, no. 5-6, pp. 401-408, 2003.

[3] S. A. El-Wakil, A. Elgarayhi, and A. Elhanbaly, "Exact periodic wave solutions for some nonlinear partial differential equations," Chaos, Solitons and Fractals, vol. 29, no. 4, pp. 1037-1044, 2006.

[4] W. R. Schowalter, "Mechanics of Non-Newtonian Fluids," Pergamon, New York, NY, USA, 1978.

[5] G. Saccomandi, "Group properties and invariant solutions of plane micropolar flows," International Journal of Engineering Science, vol. 29, no. 5, pp. 645-648, 1991.

[6] G. Saccomandi, "Some exact pseudo-plane solutions of the first kind for the Navier-Stokes equations," Zeitschrift für Angewandte Mathematik und Physik, vol. 45, no. 6, pp. 978-985, 1994.

[7] A. Kacou, K. R. Rajagopal, and A. Z. Szeri, "Flow of a fluid of the differential type in a journal bearing," Journal of Tribology, vol. 109, no. 1, pp. 100-107, 1987.

[8] C. Bridges, S. Karra, and K. R. Rajagopal, "On modeling the response of the synovial fluid: unsteady flow of a shearthinning, chemically-reacting fluid mixture," Computers \& Mathematics with Applications, vol. 60, no. 8, pp. 2333-2349, 2010.

[9] A. Farina, A. Fasano, L. Fusi, and K. R. Rajagopal, "On the dynamics of an elastic-rigid material," Advances in Mathematical Sciences and Applications, vol. 20, no. 1, pp. 193-217, 2010.

[10] A. Z. Szeri and K. R. Rajagopal, "Flow of a non-Newtonian fluid between heated parallel plates," International Journal of NonLinear Mechanics, vol. 20, no. 2, pp. 91-101, 1985.

[11] T. Aziz and F. M. Mahomed, "Closed-form solutions for a nonlinear partial differential equation arising in the study of a fourth grade fluid model," Journal of Applied Mathematics, vol. 2012, Article ID 931587, 16 pages, 2012.

[12] T. Aziz, F. M. Mahomed, and A. Aziz, "Group invariant solutions for the unsteady MHD flow of a third grade fluid in a porous medium," International Journal of Non-Linear Mechanics, vol. 47, no. 7, pp. 792-798, 2012.

[13] E. Momoniat, D. P. Mason, and F. M. Mahomed, "Non-linear diffusion of an axisymmetric thin liquid drop: group-invariant solution and conservation law," International Journal of NonLinear Mechanics, vol. 36, no. 6, pp. 879-885, 2001.

[14] V. Marinca, N. Herişanu, C. Bota, and B. Marinca, "An optimal homotopy asymptotic method applied to the steady flow of a fourth-grade fluid past a porous plate," Applied Mathematics Letters, vol. 22, no. 2, pp. 245-251, 2009.

[15] R. S. Rivlin and J. L. Ericksen, "Stress-deformation relations for isotropic materials," Journal of Rational Mechanics and Analysis, vol. 4, pp. 323-425, 1955.

[16] C. Fetecau, T. Hayat, C. Fetecau, and N. Ali, "Unsteady flow of a second grade fluid between two side walls perpendicular to a plate," Nonlinear Analysis. Real World Applications. An International Multidisciplinary Journal, vol. 9, no. 3, pp. 12361252, 2008.

[17] C. Fetecau, C. Fetecau, and M. Rana, "General solutions for the unsteady flow of second-grade fluids over an infinite plate that applies arbitrary shear to the fluid," Zeitschrift für Naturforschung A, vol. 66, pp. 753-759, 2011.

[18] M. Jamil, A. Rauf, C. Fetecau, and N. A. Khan, "Helical flows of second grade fluid due to constantly accelerated shear stresses," Communications in Nonlinear Science and Numerical Simulation, vol. 16, no. 4, pp. 1959-1969, 2011.

[19] M. Nazar, C. Fetecau, D. Vieru, and C. Fetecau, "New exact solutions corresponding to the second problem of Stokes for second grade fluids," Nonlinear Analysis. Real World Applications. An International Multidisciplinary Journal, vol. 11, no. 1, pp. 584591, 2010.

[20] F. T. Akyildiz, H. Bellout, and K. Vajravelu, "Exact solutions of nonlinear differential equations arising in third grade fluid flows," International Journal of Non-Linear Mechanics, vol. 39, no. 10, pp. 1571-1578, 2004.

[21] M. Yürüsoy and M. Pakdemirli, "Approximate analytical solutions for the flow of a third-grade fluid in a pipe," International Journal of Non-Linear Mechanics, vol. 37, no. 2, pp. 187-195, 2002.

[22] A. Aziz and T. Aziz, "MHD flow of a third grade fluid in a porous half space with plate suction or injection: an analytical approach," Applied Mathematics and Computation, vol. 218, no. 21, pp. 10443-10453, 2012.

[23] P. D. Ariel, "Flow of a third grade fluid through a porous flat channel," International Journal of Engineering Science, vol. 41, no. 11, pp. 1267-1285, 2003.

[24] R. L. Fosdick and K. R. Rajagopal, "Thermodynamics and stability of fluids of third grade," Proceedings of the Royal Society London A, vol. 369, no. 1738, pp. 351-377, 1980.

[25] G. W. Bluman and S. Kumei, Symmetries and Differential Equations, Springer, New York, NY, USA, 1989.

[26] N. H. Ibragimov, CRC Handbook of Lie Group Analysis of Differential Equations, vol. 3, CRC Press, Boca Raton, Fla, USA, 1996. 


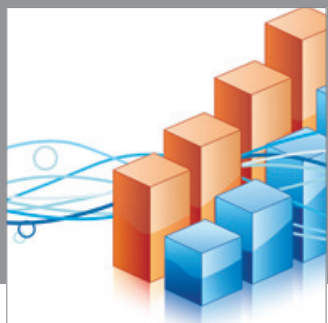

Advances in

Operations Research

mansans

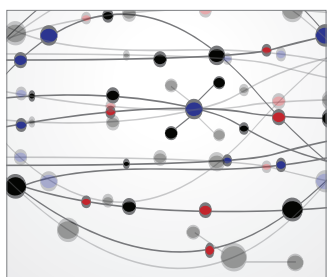

The Scientific World Journal
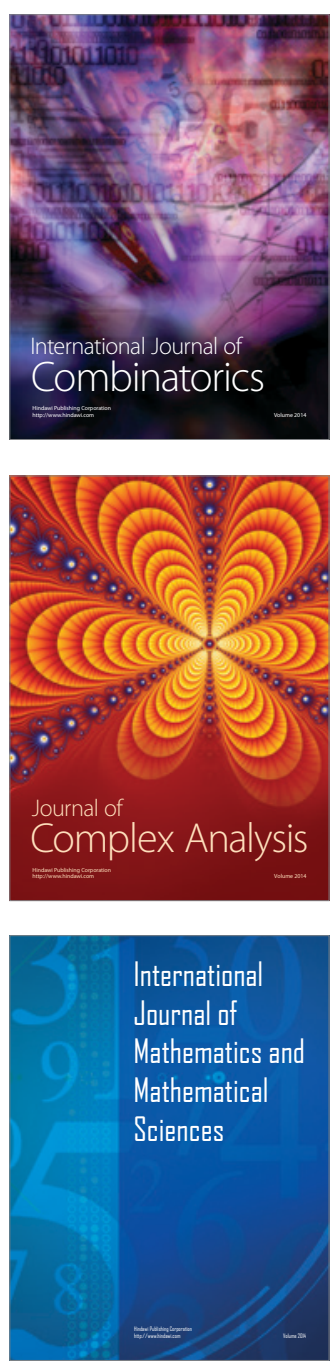
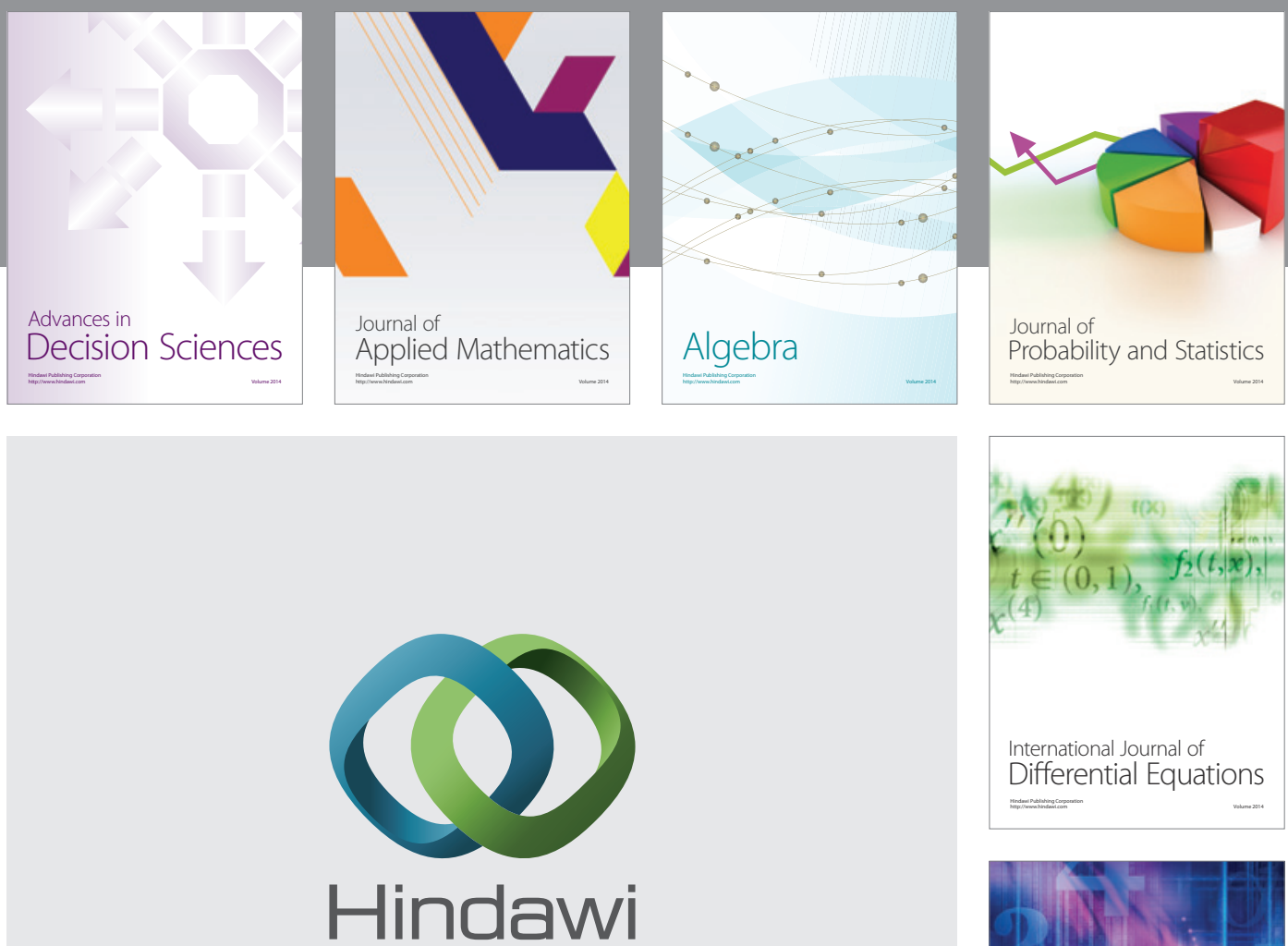

Submit your manuscripts at http://www.hindawi.com
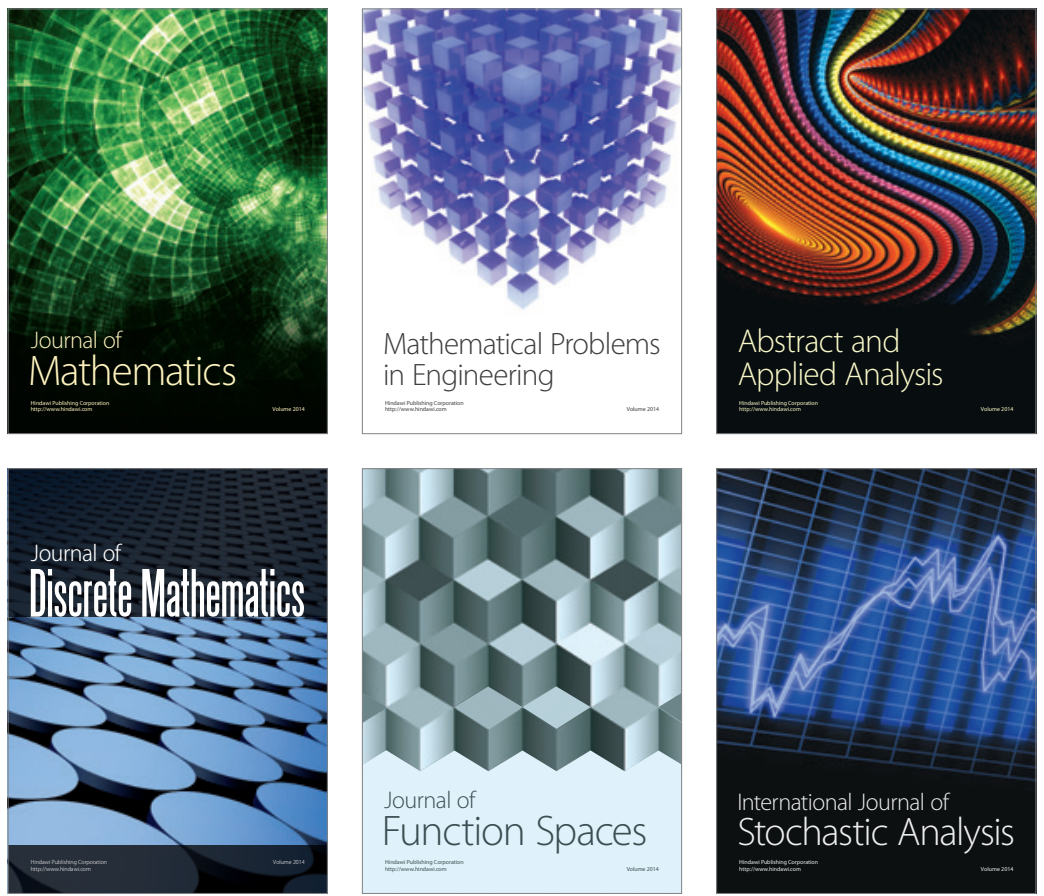

Journal of

Function Spaces

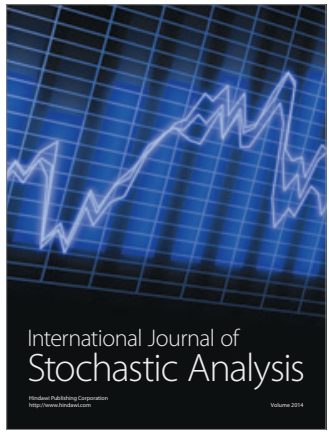

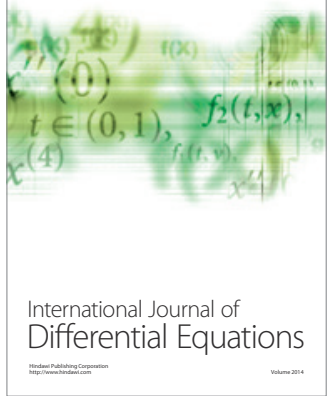
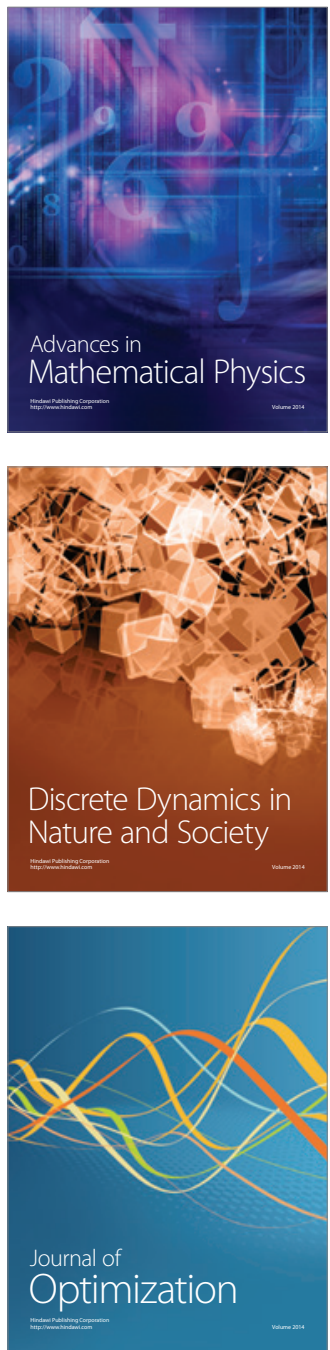